\title{
Hat der Arzt ausgedient?
}

\section{Werner Schneiter ${ }^{a}$ \\ unter Verwendung des Referates von Peter Tschudi ${ }^{b}$}

anlässlich der Veranstaltung der Ärztegesellschaft des Kantons Zürich (AGZ) vom 16. November 2005, Universität Zürich Irchel

a Kommunikationsbeauftragter der AGZ

b Dr. med., FMH Allgemeinmedizin, Vorstandsmitglied der AGZ

Korrespondenz:

Werner Schneiter

Schneiter \& Partner AG

Management \& Communication

Consultants

Dorfstrasse 71

CH-8126 Zumikon

schneiter.komm@bluewin.ch
Die Ansprüche an den Arzt von seiten der Politik, der Gesundheitsökonomen, der Krankenkassen, der Medien, der Verwaltungen, der eigenen Standesorganisationen, aber auch der Patientinnen und Patienten wachsen unaufhörlich. Die Administration nimmt absurde Ausmasse an. Viele Forderungen der Politik und der Verwaltungen sind praxisuntauglich und weitgehend sinnlos. Die Beschimpfungen in den Medien sind alltäglich, kurz: Erwartungen und Wirklichkeit klaffen immer mehr auseinander. Der nichtärztliche Beobachter fasst seine Eindrücke in den nachfolgenden Thesen zusammen.

\section{Der Arzt hat ausgedient,}

- wenn er sich durch diese Ansprüche von seiner Kernaufgabe ablenken lässt, die ein uneingeschränktes Engagement für das Wohl seiner Patienten beinhaltet;

- wenn er deswegen resigniert und «innerlich kündigt»;

- wenn er sich nur zähneknirschend weiterbildet, weil es vorgeschrieben ist;

- wenn er nur noch «Dienst nach Vorschrift» leistet und eine geregelte Arbeitszeit will;

- wenn er sich als Erfüllungsgehilfe der Krankenkassen missbrauchen lässt;

- wenn ihm der Taxpunktwert wichtiger wird als der Patient;

- wenn er seine Patientinnen und Patienten als «Krankengut» sieht und nicht als Menschen akzeptiert und respektiert.

\section{Der Arzt hat nicht ausgedient,}

- wenn er um die erkrankten Organe herum den Menschen erkennt;

- wenn er sich soviel Zeit nimmt, bis der Patient sich ernstgenommen und verstanden fühlt;

- wenn er sich bewusst ist, dass er nicht alles weiss und deshalb Kollegen fragt, mit diesen zusammenarbeitet und sie in seine Entscheidungen einbindet;

- wenn er sich immer wieder fragt, ob er die verordnete Therapie auch seinen Lieben empfehlen würde;

- wenn er sich bewusst ist, dass Angst Begleiter des Patienten ist, dieser deshalb einfühlsam betreut werden muss und dass er nur so das notwendige Vertrauen entwickeln und erhalten kann;

- wenn er sich durch die unzähligen Rezepte, welche Gesundheitspolitiker und -ökonomen sowie Kassenfunktionäre und einige Journalisten zur Senkung der Gesundheitskosten und der Qualitätssicherung verkünden, nicht kleinkriegen lässt;

- wenn er sich bemüht, bestens aus- und weitergebildet zu sein. Er kann deshalb kompetent und sicher agieren, dies im Bewusstsein, dass gute Medizin ohne Ärztinnen und Ärzte nicht stattfindet;

- wenn er bereit ist, zu seinen Entscheidungen zu stehen, auch dann, wenn sie fehlerhaft waren und zu Konsequenzen führen;

- wenn sein Patient darauf vertrauen kann, dass er für ihn jederzeit sein Bestes gibt.

Ärzte gibt und braucht es, seit und solange es Menschen gibt. 